\title{
Scrivere a mano
}

\section{Rosario Coluccia}

PUBBLICATO: 25 NOVEMBER 2020

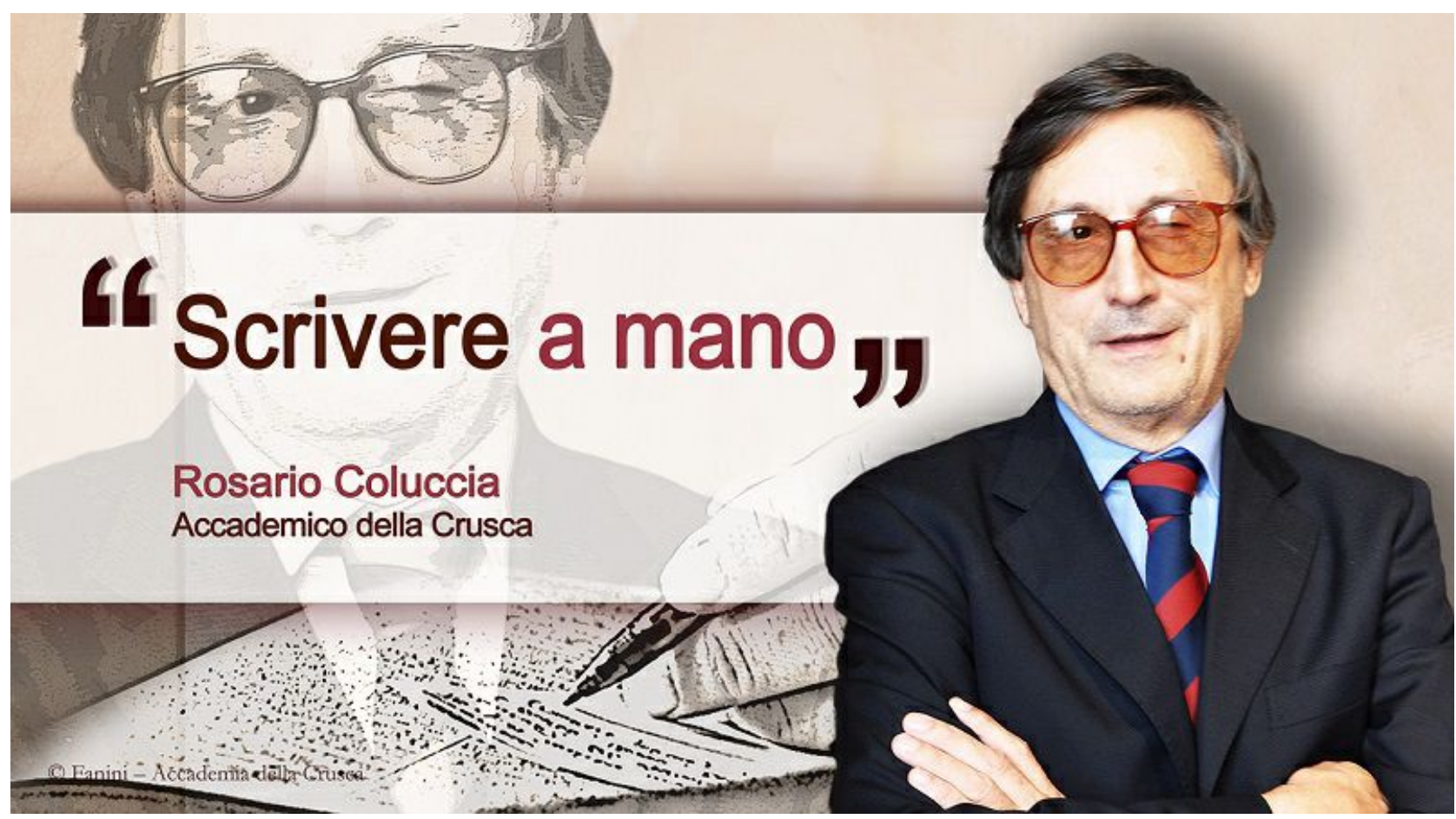

1cune settimane fa è circolata in rete una petizione, indirizzata alla ministra Azzolina in occasione dei rientro in classe, che tocca un argomento a prima vista marginale:
"Promuoviamo la bellezza della scrittura a mano", si intitola la petizione. I promotori partono da una considerazione pratica, quella della configurazione dei nuovi banchi monoposto di cui le scuole si sono dotate quest'anno. I nuovi banchi sono di dimensioni ridotte per occupare meno spazio e anche per favorire l'utilizzo di pe e di tablet. Qui può annidarsi un rischio. "Pur ritenendo che il processo di digitalizzazione della Scuola italiana, e più in generale del Paese, sia indispensabile, siamo assolutamente convinti che la scrittura a mano dei nostri studenti debba essere, nella sua straordinaria bellezza, stimolata il più possibile. È scientificamente provato, infatti, che la scrittura a mano, soprattutto in corsivo, produca enormi benefici per lo sviluppo cognitivo nell'età dell'infanzia perché accende nel bambino aree del cervello deputate al pensiero, al linguaggio, alla manualità e alla memoria". Per concludere: "Chiediamo per questo al Ministro dell'Istruzione Lucia Azzolina e agli insegnanti di ogni ordine e grado della Scuola pubblica e privata italiana il massimo impegno affinché l'utilizzo della scrittura a mano sia promosso, favorito e incoraggiato".

Al di là del titolo che vuol essere seducente, la petizione tocca contenuti di vasta portata. Non è una sollecitazione un po' fatua alla "bellezza", parola sempre più spesso usata a caso, senza specificarne i contenuti, applicata a oggetti e situazioni diversissimi, evocata con le finalità più varie. Non è neanche un complessivo nostalgico invito a ripristinare nella scuola del ventunesimo secolo pratiche didattiche del passato. Fino alla scuola degli anni '6o del Novecento bambini e ragazzi si sono sempre esercitati nella "bella scrittura". Lora di calligrafia era inserita fra le materie di studio; poi fu abbandonata, giudicata strumento educativo sorpassato, mortificante della creatività. 
Un dato, per quanto esterno, pare difficilmente contestabile. Gli studenti dei decenni passati per la maggior parte erano in condizione di produrre temi, riassunti e diari con nitidezza e pulizia quasi tipografiche. Meno gradevole la forma esterna dei testi elaborati da gran parte dei ragazzi di oggi. Da anni gli insegnanti della scuola primaria e media segnalano la crescente difficoltà dei loro allievi a scrivere manualmente. Nei testi redatti a mano i caratteri appaiono incerti e disallineati, con parole mal disposte sul rigo, con i tratti delle singole lettere a volte difficili da decifrare, con vacillanti legamenti tra una lettera e l'altra, con incongrui miscugli di stili e di caratteri nelle stesse parole o nella stessa sequenza di parole: corsivo e stampatello, maiuscolo e minuscolo. Non vale solo per i bambini delle elementari o al massimo delle medie. La difficoltà di scrivere a mano è presente in adolescenti delle scuole secondarie superiori e coinvolge in maniera preoccupante i giovani universitari. Spesso gli scritti manuali degli studenti medi e universitari rasentano l'indecifrabilità, con pensieri sconclusionati, in una forma che non rispetta gli standard minimi di coerenza e coesione.

L'aspirazione a una scrittura ordinata e ben leggibile non è un fatto estetizzante. La scarsa connessione neuro-cerebrale tra pensiero e manualità crea ritardi nello sviluppo del linguaggio, parlato e scritto. $\mathrm{Ne}$ viene coinvolto il processo cognitivo di bambini e adolescenti, fondamentale perché implica l'esercizio di una capacità umana molto antica (la scrittura è stata inventata più o meno cinquemila o cinquemilacinquecento anni fa), che oggi corriamo il rischio di perdere. Diciamolo in maniera esplicita. La scrittura a mano non può essere sostituita dalla scrittura su tastiera, sono entrambe utili perché assolvono a funzioni diverse. Nel mondo occidentale bambini e ragazzi sono fortemente sedentarizzati; alcuni non sanno abbottonarsi i vestisti o allacciarsi le scarpe (sono in gran voga scarpe senza lacci, definite "a strappo" o "con strappi"; praticissime, assicura la pubblicità, e crescono le vendite delle scarpe a strappo); altri non sanno lavarsi i denti da soli; altri non riescono a fare operazioni semplici (tracciare cerchi e rettangoli con l'aiuto di compasso e di righello) o addirittura attività semplicissime (ridurre un foglio di carta in segmenti più piccoli tendenzialmente uguali). E, nello stesso tempo, mostrano carenze espressive e linguistiche. Redigere testi scritti in maniera chiara e ordinata è un eccellente allenamento cerebrale.

Una ricerca coordinata da Benedetto Vertecchi, università di Roma Tre, ha mostrato che, con opportuno allenamento alla scrittura manuale, bambini di terza, quarta e quinta elementare, migliorano progressivamente la qualità grafica dei loro testi e nello stesso tempo ottengono una maggiore appropriatezza ortografica e una più accurata selezione del lessico. A livello cerebrale esiste un legame tra attività manuale e area del linguaggio, che si influenzano reciprocamente. Nel tracciare manualmente i caratteri del corsivo al cervello del bambino è richiesto uno sforzo in più, la forma di ciascuna lettera deve essere continuamente plasmata perché sia possibile legarla alle altre. Si tratta di una sfida che non è presente nel carattere stampatello o quando si adoperano strumenti elettronici come il touchscreen, che richiedono una gestualità semplice e ripetitiva.

La difficoltà di scrivere nitidamente ha riflessi sulla qualità dell'apprendimento e sulla capacità di coordinare il pensiero. La caduta investe sia la capacità di tracciare adeguatamente i caratteri sul foglio, sia quella di organizzare correttamente la sequenza di parole e le frasi necessarie per trasmettere il messaggio. Mettiamo per ipotesi che nessuno scriva più con carta e penna, che si usino solo mezzi digitali. Il correttore automatico riduce la consapevolezza ortografica: non c'e bisogno di conoscere l'ortografia delle parole, il correttore automatico vi provvede al posto nostro. C'è di più. Il ricorso ossessivo alla funzione "copia e incolla" riduce la necessità di sviluppare una linea argomentativa coerente. La procedura fu inventata nel 1973 da Lawrence Gordon Tesler (morto nel febbraio 2020), ricercatore della Xerox a Palo Alto, con lo scopo a prima vista meramente strumentale e pratico, quasi banale, di risparmiare a chi digita la fatica di riscrivere manualmente parole o frasi. Invece ha avuto una portata dirompente, ha influito sulle strutture mentali di chi elabora un testo, ha cambiato la 
maniera di pensare e di fare ricerca, trasformando il modo in cui oggi vengono percepiti la organicità di un testo e concetti quali la ripetizione e il plagio. Prima che esistesse il "copia e incolla", appropriarsi di un testo altrui e includerlo nel proprio (operazione dolosa) richiedeva la riscrittura a mano o a macchina del brano copiato e comportava almeno un certo impegno intellettuale: non era possibile riscrivere qualcosa senza comprenderlo abbastanza profondamente. Oggi non è così. Nel web ci sono miliardi di pagine che non sono altro che la copia di altre pagine, in una sequenza senza storia, senza origine e senza fine. Si può copiare qualsiasi cosa senza interrogarsi sulla sua plausibilità, senza nemmeno sforzarsi di conoscerne a fondo il significato, è sufficiente uno sguardo alla prima riga o alle prime parole, per assicurarsi di non essere del tutto fuori strada. Al pari di ogni altra invenzione, quella di Tesler è utile e pericolosa nello stesso tempo. Sicuramente non va criminalizzata a priori. "Copia e incolla responsabilmente" potrebbe essere un buon motto. E verifica sempre. La tecnologia abitua a pensare che c'e sempre una risposta all'esterno e non nella nostra testa. Ne consegue l'abbandono del discernimento e la caduta verticale della memoria: perché memorizzare una data, un nome, un verso? Cerco nella rete, lì c'è tutto.

Torniamo al punto da cui siamo partiti. Il recupero della scrittura a mano è un obiettivo importante, anzi importantissimo, perché va ben al di là della chiarezza (a fini interpretativi) delle scritture. (Che non è poco). Lo ha ribadito, in più occasioni, Francesco Sabatini, presidente onorario dell'Accademia della Crusca, le cui argomentazioni provo a riassumere nelle righe seguenti. Non si tratta di una sorta di mania passatista dei "letterati", è tesi sostenuta su basi scientifiche dai neurologi. Uno di questi (Leonardo Fogassi) ha ribadito di recente che "l'area più ampia e sviluppata della corteccia cerebrale è quella collegata ai movimenti più fini: quelli compiuti dalle mani e dalla bocca". Le mani sono responsabili di infinite attività, compresa quella della scrittura accurata. La bocca produce i movimenti articolatori compiuti per parlare, che devono essere ben marcati: l'immagine sonora si fissa nella memoria. Sabatini mi invita a divulgare un'immagine che ha disegnato un neurologo canadese (Wilder Penfield) mezzo secolo fa, una delle variazioni del suo Homunculus: mani e bocca sono enormi, testimoniano il ruolo centrale che questi organi ricoprono nello sviluppo della storia dell'uomo. Evidenze sconosciute nella cultura comune e, quasi sempre, ignorate da coloro che operano nella scuola.

Tocca a noi diffondere questi contenuti innovativi, gli esiti saranno benefici. Incoraggiando la scrittura manuale (senza rinunziare alle opportunità del digitale) la nostra Accademia riafferma il suo compito statutario: sostenere la lingua italiana e promuoverne lo studio e la conoscenza. Il recupero della scrittura a mano merita grande attenzione. Senza demonizzare pc, tablet e smartphone che devono affiancare, non sostituire, la modalità tradizionale di scrittura. Vecchio e nuovo possono convivere, non sono in contrasto, l'uno non esclude l'altro. Accostiamoci al nuovo senza rinunziare al vecchio, è questa la sfida.

\section{Cita come:}

Rosario Coluccia, Scrivere a mano "Italiano digitale", 2020, XV, 2020/4 (ottobre-dicembre) DOI: $10.35948 / 2532-9006 / 2020.5428$

Copyright 2020 Accademia della Crusca

Pubblicato con licenza creative commons CC BY-NC-ND 